\title{
Clinical spectrum of intrathoracic Castleman disease: a retrospective analysis of 48 cases in a single Chinese hospital
}

Jin mei Luo ${ }^{1 \dagger}$, Shan $\mathrm{Li}^{1 \dagger}$, Hui Huang ${ }^{1 *}$, Jian $\mathrm{CaO}^{2}$, Kai Xu² , Ya lan Bi ${ }^{3}$, Rui e Feng ${ }^{3}$, Cheng Huang ${ }^{4}$, Ying zhi Qin ${ }^{4}$, Zuo jun $\mathrm{Xu}^{1}$ and Yi Xiao ${ }^{1}$

\begin{abstract}
Background: Thorax is the common place to develop Castleman disease (CD), but there is no systemic clinical analysis for intrathoracic CD.

Methods: We conducted a retrospective analysis of 48 intrathoracic CD patients with definite pathological diagnosis who were hospitalized between 1992 and 2012 in a Chinese tertiary referral hospital.

Results: The study included 16 cases with unicentric CD (UCD) and 32 cases with multicentric CD (MCD). UCD were younger than MCD (30.5y vs 41.6ys, $P<0.05)$. MCD were more symptomatic $(50 \%$ vs $96.9 \%, P<0.001)$ and sicker than UCD, including more fever, hepatomegaly and/or splenomegaly and hypoalbuminemia. All of UCD showed solitary mass in various sites and two of them were complicated by small pleural effusion. In the MCD group, their chest CT showed obvious lymphadenopathy in the hilum and/or mediastinum (100\%), diffuse parenchymal lung shadows (43.75\%), pleural effusion (40.6\%), mass in the mediastinum (6.25\%) or hilum (3.12\%) and bronchiolitis obliterans (BO) (3.12\%). Besides LIP-like images, multiple nodules of different size and sites, patchy, ground-glass opacities and consolidation were showed in their chest $\mathrm{CT}$. Surgery were arranged for all UCD for diagnosis and treatment and all were alive. In MCD group, superficial lymph nodes biopsies (21 cases), surgery biopsy (9 cases) and CT-guided percutaneous lung biopsy (2 cases) were performed. Hyaline vascular (HV) variant were more common in the UCD group (75\% vs 37.5\%, P < 0.05). In MCD group, 28 cases were prescribed with chemotherapy, one refused to receive therapy and the rest three were arranged for regular follow-up. Among MCD, 18 cases was improved, 7 cases was stable, 4 cases lost follow-up and 3 cases died.
\end{abstract}

Conclusions: Intrathoracic MCD was more common than UCD in our hospital. MCD was older, more symptomic and sicker than UCD. HV variant were more common in UCD. All of UCD showed mass in various intrathoracic locations and surgery resection was performed for all and all were alive. Mass, pleural effusion, $\mathrm{BO}$ and diffuse pulmonary shadows, including LIP-like images, multiple nodules of different size and sites, patchy, GGO and consolidations were showed in our MCD. Most of MCD cases were arranged with chemotherapy and their prognosis were worse than UCD's.

Keywords: Castleman disease, Intrathoracic, Unicentric, Multicentric, Clinical characters

\footnotetext{
* Correspondence: pumchhh@126.com

${ }^{\dagger}$ Equal contributors

'Department of Respiratory Medicine, Peking Union Medical College Hospital, Chinese Academy of Medical Sciences \& Peking Union Medical College, \#1 Shuaifuyuan Street, Dongcheng District, Beijing 100730, China Full list of author information is available at the end of the article
} 


\section{Background}

Castleman disease (CD) is also known as angiofollicular hyperplasia or giant lymph node hyperplasia. It can be classified as unicentric (unifocal or localized) or multicentric (multifocal or generalized) according to the lesions involved [1-3]. Although CD can occur at any site where lymphoid tissue is normally present, approximately $70 \%$ of $C D$ occurred in the thorax $[4,5]$. Intrathoracic involvement of $\mathrm{CD}$ can manifest as a mediastinal and/or hilar mass, pleural mass, tracheal mass, pleural effusion, bronchiolitis obliterans, diffuse parenchymal lung disease, etc. [6-16]. However, most of this data comes from rare case reports or smaller case series. And some of these included cases with the main focus of disease in the neck, supraclavicular and/or axillary nodes [6]. Here we describe the clinical and radiological findings, as well as the outcomes of 48 intrathoracic CD patients in a single Chinese tertiary-referral hospital.

\section{Methods \\ Patients}

Using a computer-assisted search for patients who were hospitalized with CD at Peking Union Medical College Hospital from January 1992 to December 2012, we identified $151 \mathrm{CD}$ cases with the confirmed pathological diagnosis. Patients with POEMS syndrome (polyneuropathy, organomegaly, endocrinopathy, M-protein, skin pigmentation) and without complete clinical or radiological data (32 cases) were excluded even if they had the typical pathological features of $\mathrm{CD}$. After review of the medical records, radiologic images and pathological manifestations, 48 intrathoracic $\mathrm{CD}$ cases were identified. All of the patients had the main focus of disease inside the chest cage, excluding those with their main involvements in the neck, supraclavicular region, axillary nodes, heart, pericardium and around the aorta. The remaining 71 cases were classified as the extrathoracic CD group.

The following information was recorded for analysis: age, sex, symptoms at presentation, physical examination, and laboratory findings (including serological results, radiologic findings, and pathological results), treatment and outcomes. Patients' computed tomography (CT) images were downloaded from our hospital's image data bank. Two radiologists conducted a consensus reading of the $\mathrm{CT}$ images. All of the cases with diffuse pulmonary disease were admitted after 1998, so all of these patients had high-resolution chest $\mathrm{CT}$ imaging. The biopsies from sections of involved tissues and/or organs were reviewed by two pathologists in our pathological department (Ya lan Bi and Rui e Feng). The pathological diagnosis of $\mathrm{CD}$ was established according to Flendrig and Keller's criteria in all of the enrolled patients $[17,18]$. The hyaline vascular (HV) variant is characterized by the presence of atrophic germinal centers which are surrounded by expanded mantle zones of small lymphocytes forming concentric rings ("Onion-skinning"), and hyalinized blood vessels penetrating into follicles. For the plasma cell (PC) variant, there is retained lymph node architecture and variable germinal center hyperplasia, with expanded mantle zones and a marked plasmacytosis in the mantle or paracortical regions. Malignant lymphoma, granulomas and histiocytic necrotizing lymphadnitis are excluded. Immunostaining with CD3 and CD20 were performed for both HV and PC variant, but immunostaining with $\mathrm{CD} 38$ and $\mathrm{CD} 138$ were performed only for PC variant. The mixed variant displays features of both $\mathrm{HV}$ and $\mathrm{PC}$ variant.

Other definitions in this study included : (1) anemia defined as hemoglobin $<110 \mathrm{~g} / \mathrm{l}$ for female and $<120 \mathrm{~g} / \mathrm{l}$ for male, (2) thrombocytopenia defined as PLT $<100 \times 10^{9} / \mathrm{L}$, (3) hypoalbuminemia defined as serum albumin $<35 \mathrm{~g} / \mathrm{l}$, (4) elevation of immunoglobin G(IgG) defined as serum IgG $>17 \mathrm{~g} / \mathrm{L}$, and (5) elevation of lactate dehydrogenase (LDH) defined as serum LDH $>270 \mathrm{U} / \mathrm{L}$.

All patient data were followed to September 2014. Data was collected through telephone, letters and cases records. All patients and/or their families provided written informed consent to publish their clinical details. The study was approved by the ethics committee of Peking Union Medical College Hospital.

\section{Statistical analysis}

Data was analyzed using the Statistical Analysis System (SAS) version 8.0 software package. Quantitative variables were described using mean $\pm \mathrm{SD}$ and categorical data using frequency and percentage in the text and figures. A comparison between two groups was analyzed by the student's t-test when data was normally distributed. The Kruskal-Wallis test was used when data were not normally distributed. A value of $\mathrm{P}<0.05$ was considered to be statistically significant.

\section{Results}

Human immunodeficiency virus (HIV) antibody screening test was arranged for $95 \mathrm{CD}$ cases, including 42 intrathoracic cases, and all of them were negative. All cases which were not screened for HIV antibodies were admitted before 2000 .

\section{Demographic characteristics}

The intrathoracic CD group consisted of 19 male and 29 female patients with a mean age of 37.9y (range between 13-67 years of age). Among them, 16cases (33.3\%) were classified with unicentric CD (UCD) and the remaining 32cases $(66.7 \%)$ were diagnosed with muticentric $C D$ (MCD). As we know now that there were many differences between the UCD and MCD in clinical, radiological, pathological and prognostic features $[1,19,20]$, 
we compared the intrathoracic UCD and MCD cases in our hospital (Table 1).

UCD patients were younger than MCD cases, with the mean age of $30.5 \mathrm{y}$ and $41.6 \mathrm{ys}$, respectively $(\mathrm{t}=3.03$, $\mathrm{P}=0.004)$. But there was no difference in the gender distribution between them, with male cases of $50 \%$ vs $34.4 \%$, respectively $\left(x^{2}=1.09, P=0.30\right)$ (Table 1$)$.

\section{Clinical and radiological manifestations}

The clinical characteristics are summarized in Table 1 . Half of the UCD cases had no obvious symptoms when they were admitted to our hospital, but were found to have masses on a routine chest radiographic examination.

Table 1 Differences between unicentric and multicentric intrathoracic CD cases

\begin{tabular}{|c|c|c|c|}
\hline & UCD & MCD & $\mathbf{P}$ \\
\hline Age (mean, years) & $30.44 \pm 8.00$ & $41.56 \pm 13.50$ & 0.004 \\
\hline \multicolumn{4}{|l|}{ Gender } \\
\hline Male & $8 / 50 \%$ & $11 / 34.4 \%$ & 0.2967 \\
\hline Female & $8 / 50 \%$ & $21 / 65.6 \%$ & \\
\hline \multicolumn{4}{|l|}{ Pathological type } \\
\hline Hyaline vascular & $3 / 18.75 \%$ & $16 / 50 \%$ & \\
\hline Plasma cell & $12 / 75 \%$ & $12 / 37.5 \%$ & 0.049 \\
\hline Mixed & $1 / 6.25 \%$ & $4 / 12.5 \%$ & \\
\hline Non-symptomatic & $8 / 50 \%$ & $1 / 3.1 \%$ & $<0.001$ \\
\hline Fever & 0 & $16 / 50 \%$ & $<0.001$ \\
\hline Pleural effusion & $2 / 12.5 \%$ & $13 / 40.6 \%$ & 0.0475 \\
\hline $\begin{array}{l}\text { Hepatomegaly and/or } \\
\text { splenomegaly }\end{array}$ & 0 & $16 / 50 \%$ & $<0.001$ \\
\hline Anemia & $3 / 18.8 \%$ & $15 / 46.9 \%$ & 0.0578 \\
\hline Hemoglobin (g/l) & $133.13 \pm 29.70$ & $108.66 \pm 26.60$ & 0.0059 \\
\hline \multicolumn{4}{|l|}{ Platelet $\left(\times 10^{9} / \mathrm{I}\right)$} \\
\hline$>300 \times 10^{9} / I$ & $4 / 25 \%$ & $12 / 37.5 \%$ & 0.6785 \\
\hline$<100 \times 10^{9} / 1$ & $1 / 8.3 \%$ & $2 / 10 \%$ & \\
\hline Hypoalbuminia & $4 / 25 \%$ & $22 / 68.8 \%$ & 0.0041 \\
\hline Elevation of serum $\mathrm{LDH}^{\ddagger}$ & $5 / 30 \%$ & $5 / 16.1 \%$ & 0.6731 \\
\hline \multicolumn{4}{|l|}{ HIV-Ab } \\
\hline Not done & 0 & $6 / 18.75 \%$ & \\
\hline Negative & $16 / 100 \%$ & $26 / 100 \%(n=26)$ & 1 \\
\hline Chemotherapy cases & 0 & $28 / 87.5 \%$ & $<0.001$ \\
\hline \multicolumn{4}{|l|}{ Outcomes } \\
\hline Cured & $16 / 100 \%$ & 0 & \\
\hline Improved & 0 & $18 / 56.3 \%$ & \\
\hline Stable & 0 & 7/21.9\% & $<0.001$ \\
\hline Died & 0 & $3 / 9.4 \%$ & \\
\hline Lost follow-up & 0 & $4 / 12.5 \%$ & \\
\hline
\end{tabular}

${ }^{\ddagger}$ Fifteen UCD cases and 31 MCD cases were detected with serum LDH. LDH: lactate dehydrogenase, HIV: human immunodeficiency virus.
Thirty-one out of 32 cases with MCD were symptomatic (50\% vs $96.9 \%, \chi^{2}=15.4, \mathrm{P}<0.001$ ). Although fever was the most common symptom for MCD cases (16/50\%), there were no patients with UCD presenting with fever (0 vs $50 \%, X^{2}=11.4, P<0.001$ ). None of the patients with UCD had hepatomegaly and/or splenomegaly, but half of these cases in the MCD group had hepatomegaly and/or splenomegaly ( 0 vs $\left.50 \%, X^{2}=11.4, P<0.001\right)$.

In the UCD group, 8 cases complained of at least one of the following symptoms: dyspnea or chest distress $(4 / 25 \%)$, dry cough $(3 / 18.75 \%)$, backache $(1 / 6.25 \%)$ and chest pain $(1 / 625 \%)$. Although all cases had a solitary mass in their contrast chest $\mathrm{CT}$, they were distributed in various sites: mediastinum (11 cases/68.75\%), lung hilum (3 cases/18.75\%), intrapulmonary fissure (1 case/6.25\%) and in the lung (1 case/6.25\%) (Figure 1). Punctate calcification in the mass was showed in 5 UCD cases. Fourteen cases had been arranged for the chest contrastenhanced CT, and two cases had been arranged for chest magnetic resonance imaging (MRI). High to moderate enhancement of the mass could be seen in the enhanced CT. The mean size of the mass was $113.56 \mathrm{~cm}^{3}$ (ranged from $20 \mathrm{~cm}^{3}$ to $336 \mathrm{~cm}^{3}$ ). Two of them were complicated by small pleural effusions.

In the MCD group, 31cases suffered from at least one of the following symptoms: fever $(16 / 50 \%)$, cough $(15 / 46.88 \%)$, dyspnea (15/46.88\%), fatigue (10/31.25\%), abdominal distention $(6 / 18.75 \%)$, edema $(6 / 18.75 \%)$ and chest pain $(1 / 3.12 \%)$. Only one patient had no obvious symptoms.

All cases had significant abnormalities on their chest CT (Table 2), including obvious lymphadenopathy in the hilum and/or mediastinum (32/100\%), diffuse parenchymal lung disease (14/43.75\%), pleural effusion (13/40.6\%), mass in the mediastinum $(2 / 6.25 \%)$ or hilum $(1 / 3.12 \%)$ and bronchiolitis obliterans (BO) (1/3.12\%). Thoracentesis was performed in 8 of the cases. Exudative fluid was acquired in 6 of the cases and among them, 5 cases had bilateral effusions. None of the patients had chylous effusions. All of these 6 cases also had high levels of serum IgG. But only one of the cases underwent a pleural biopsy, which showed unspecific chronic inflammation. Highresolution chest CT in 14 of the cases with parenchymal involvement showed lymphocytic interstitial pneumonia (LIP)-like changes in 3 of the cases, multiple nodules of different sizes and sites in 6 of the cases, multiple patchy infiltrates in 6 of the cases, ground-glass opacities (GGO) in 5 of the cases and consolidation in 3 cases (Figure 2).

\section{Laboratory tests}

Some of the test results are summarized in Table 1. All 48 cases had complete blood count results. In the UCD group, there were 3 cases $(18.8 \%)$ who had anemia, with a mean hemoglobin of $(133.13 \pm 29.70) \mathrm{g} / \mathrm{L}$. In the MCD 


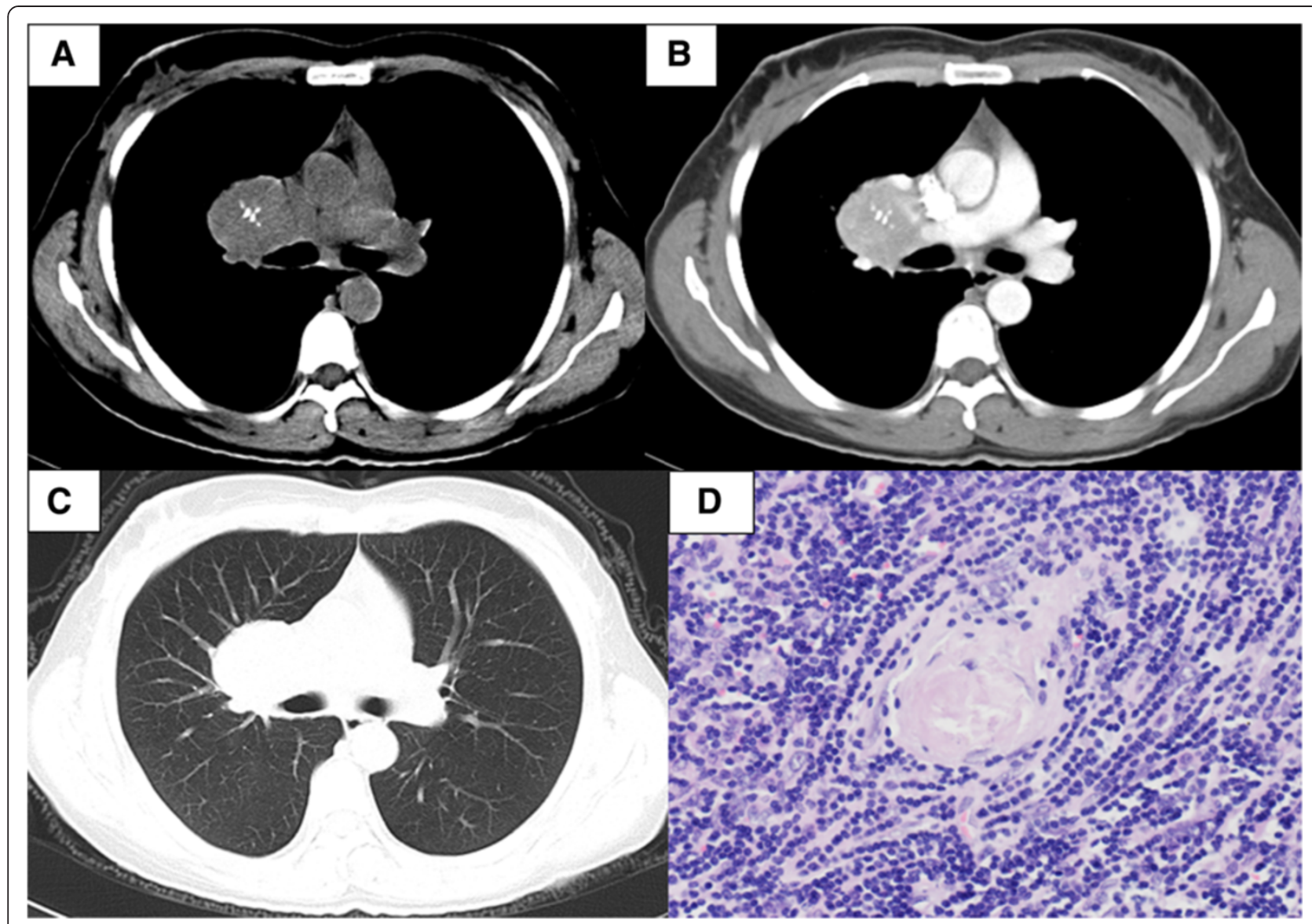

Figure 1 A 40-year-old woman was diagnosed with unicentric Castleman disease of hyaline vascular variant after thoracic surgery. She was admitted to our hospital because of slight chest distress for 9 months. Contrasted chest CT showed a well-defined and homogeneous enhanced mass in the right hilum, with coarse calcification (A-C). It was almost normal under the examination of bronscopy and the transbronchial needle aspiration (TBNA) biopsy of the right hilar mass showed chronic inflammation. Whole resection of the mass was performed and prominent diffuse proliferation of lymphoid cells with central hyalinized vascular structures was showed pathologically (D, Hematoxylin and eosin, $\times 200)$. she was diagnosed with Castleman disease of hyaline vascular variant. Chemotherapy was not suggested. she had been alive without recurrence for 7 years.

group, almost half of them (15/46.9\%) had anemia and the mean hemoglobin was $(108.66 \pm 26.60) \mathrm{g} / \mathrm{L}$. It seemed like the MCD patients were prone to suffer from anemia, but the difference was not statistically significant. There was no difference in the platelet counts between the UCD group and the MCD group.

Serum LDH level have been associated with the prognosis of some kinds of lymphoma [21]. We compared the initial serum LDH level between these two groups and it showed no difference. There were 5 cases in both groups with elevated serum LDH (30\% vs $16.1 \%, \quad Z=0.33$, $\mathrm{P}=0.67$ ). Hypoalbuminemia was more common in patients with $\mathrm{MCD}(25 \%$ vs $68.8 \%, \mathrm{Z}=8.22, \mathrm{P}=0.04)$. As only 3 cases with UCD had been arranged with serum IgG detection, the difference between them could not be compared. In the MCD group, the serum IgG was tested in 27 cases, and the mean serum IgG was $29.5 \mathrm{~g} / \mathrm{l}$. Twenty of the cases $(74.1 \%)$ showed elevated serum IgG, with the highest IgG found to be $133 \mathrm{~g} / \mathrm{l}$.
It was reported that kinds of systemic connective tissue disease could complicated by Castleman's disease [22]. Autoantibodies analysis, including antinuclear antibodies, anti-extractable nuclear antigens (ENA) antibodies, anti-neutrophil cytoplasmic antibodies (ANCA) should be tested for them. As a retrospective study, the results of autoantibodies were acquired in only 4cases with UCD and 30cases with MCD. For these 4 UCD cases, only one had positive ANA with homogeneous pattern at 1:80 titer. For the enrolled $30 \mathrm{MCD}$ cases, 5 cases had positive ANA, with speckled pattern (S), nucleolar $(\mathrm{N})$ or homogeneous pattern $(\mathrm{H})$, ie, S1:160, $\mathrm{N} 1: 40, \mathrm{H1}: 80, \mathrm{H} 1: 320$ and $\mathrm{H1:320}$. None of our enrolled 48 cases complicated by a systemic connective tissue disease.

\section{Pathological features}

All patients had a definite pathological diagnosis. Surgery was arranged for all of the 16 cases in the UCD 
Table 2 Chest CT manifestations of the enrolled 48 cases

\begin{tabular}{|c|c|}
\hline Chest CT characters & $n / \%$ \\
\hline \multicolumn{2}{|l|}{ UCDs $(n=16)$} \\
\hline \multicolumn{2}{|l|}{ Solitary mass ( $n=16,100 \%)$} \\
\hline \multicolumn{2}{|l|}{ Location } \\
\hline Mediastinum & $11 / 68.75 \%$ \\
\hline Lung hilum & $3 / 18.75 \%$ \\
\hline Intrapulmonary fissure & $1 / 6.25 \%$ \\
\hline Lung & $1 / 6.25 \%$ \\
\hline Size & $\begin{array}{l}(113.56 \pm 81.31) \mathrm{cm}^{3} \\
{\left[(20-336) \mathrm{cm}^{3}\right]}\end{array}$ \\
\hline Punctate calcification in the mass & $5 / 31.25 \%$ \\
\hline \multicolumn{2}{|l|}{$\begin{array}{l}\text { Enhancement in the contrast-enhanced } \\
\text { CT }(n=14)^{\Phi}\end{array}$} \\
\hline \multicolumn{2}{|l|}{ Pattern } \\
\hline Homogeneous & $12 / 85.71 \%$ \\
\hline Heterogeneous & $2 / 14.29 \%$ \\
\hline \multicolumn{2}{|l|}{ Degree } \\
\hline High & $13 / 92.86 \%$ \\
\hline Moderate & $1 / 7.14 \%$ \\
\hline Pleural effusion & $2 / 14.29 \%$ \\
\hline \multicolumn{2}{|l|}{ MCDs $(n=32)$} \\
\hline \multicolumn{2}{|l|}{ Lymphadenopathy } \\
\hline \multicolumn{2}{|l|}{ Location } \\
\hline $\begin{array}{l}\text { Diffuse lymphadenopathy in the hilum } \\
\text { and/or mediastinum }\end{array}$ & $32 / 100 \%$ \\
\hline Superficial lymphadenopathy & $20 / 62.5 \%$ \\
\hline Cervical LNs & $20 / 62.5 \%$ \\
\hline Superclavicular LNs & $4 / 12.5 \%$ \\
\hline Axillary LNs & $12 / 37.5 \%$ \\
\hline Diffuse parenchymal lung disease & $14 / 43.75 \%$ \\
\hline Multiple nodules in different size & $6 / 18.75 \%$ \\
\hline Multiple patchy infiltrates & $6 / 18.75 \%$ \\
\hline GGO & $5 / 15.63 \%$ \\
\hline LIP-like pattern & $3 / 9.38 \%$ \\
\hline Consolidation & $3 / 9.38 \%$ \\
\hline Bronchiolitis obliterans & $1 / 3.12 \%$ \\
\hline Mass & $3 / 9.38 \%$ \\
\hline Mediastinal mass & $2 / 6.25 \%$ \\
\hline Hilar mass & $1 / 3.12 \%$ \\
\hline Pleural effusion & $13 / 40.6 \%$ \\
\hline
\end{tabular}

${ }^{\Phi}$ Two cases with UCDs had been arranged for thoracic MRI instead of the controlled chest CT.

CT: computed tomography, LN: lymph node, GGO: ground-glass opacities, LIP: lymphocytic interstitial pneumonia.

group (Table 3). In the MCD, 21 cases were diagnosed with superficial lymph node biopsies, including cervical $(9 / 28.13 \%)$, axillary $(6 / 18.75 \%)$ and supratrochlear lymph nodes $(1 / 3.12 \%)$. Sixteen cases had at least two superficial lymph node biopsies. Eight cases (25\%) were performed with video-assisted thoracic surgery (VATS) for biopsy of mediastinal lymph nodes or lung and one case (3.12\%) underwent surgery for biopsy of lymph nodes in the retroperitoneal space. CT-guided percutaneous lung biopsies were arranged for two cases (6.25\%).

With respect to our intrathoracic respiratory CD histological type, 24 cases (50\%) were classified as the HV variant and 19 cases (39.58\%) were diagnosed with thePC variant. The remaining 5 cases $(10.42 \%)$ were diagnosed with the mixed variant form. HV variants were more common in the UCD group ( $75 \%$ vs $37.5 \%$, $\left.X^{2}=6.03, P=0.049\right)$ (Table 1 ).

\section{Treatment and prognosis}

Four cases with MCD were lost to follow-up, and the other cases were available with a mean follow-up time of 75.4 months.

All UCD cases had thoracic surgery for diagnosis and treatment. None of the cases were treated with chemotherapy and all of them were alive.

In MCD group, none of the patients had therapeutic surgery performed, butexploratory surgery for biopsy were arranged for them to acquire pathological diagnosis. Twenty-eight of the 32 cases with MCD were prescribed chemotherapy: CHOP (cyclophosphamide, adriamycin, vincristine and corticosteroid 20cases), COP (cyclophosphamide, vincristine and corticosteroid, 1case), R-CHOP (rituximab and CHOP, 1 case), MP (melphala plus prednisone, 1 case), melphala plus thalidomide and prednisone (1 cases) and corticosteroids plus cyclophosphamide (4 cases). For the remaining $4 \mathrm{MCD}$ cases, 3 of the cases were suggested for regular follow up because of mild symptoms with steady medication for at least 3 years, and the last one refused chemotherapy but complained of superficial lymphadenopathy at multiple sites for 11 years and exertional dyspnea for 2 years (Figure 2).

In MCD group, three cases (9.38\%) died: one was complicated by $\mathrm{BO}$ and paraneoplastic pemphigus (PNP) and she died of respiratory failure, one had diffuse parenchymal lung disease and suffered from a severe lung infection during the chemotherapy and died, and the other was complicated by myocardial amyloidosis and pulmonary hypertension. All of them were prescribed chemotherapy. Four cases were lost to follow-up after they were discharged from our hospital. Seven of the cases were stable and 18 cases improved after discharge.

\section{Discussion}

Although CD could arise from any site where lymphoid tissue is normally present, the chest was the most common place to develop CD [4-6]. There were several studies which reported thoracic and/or pulmonary CD 


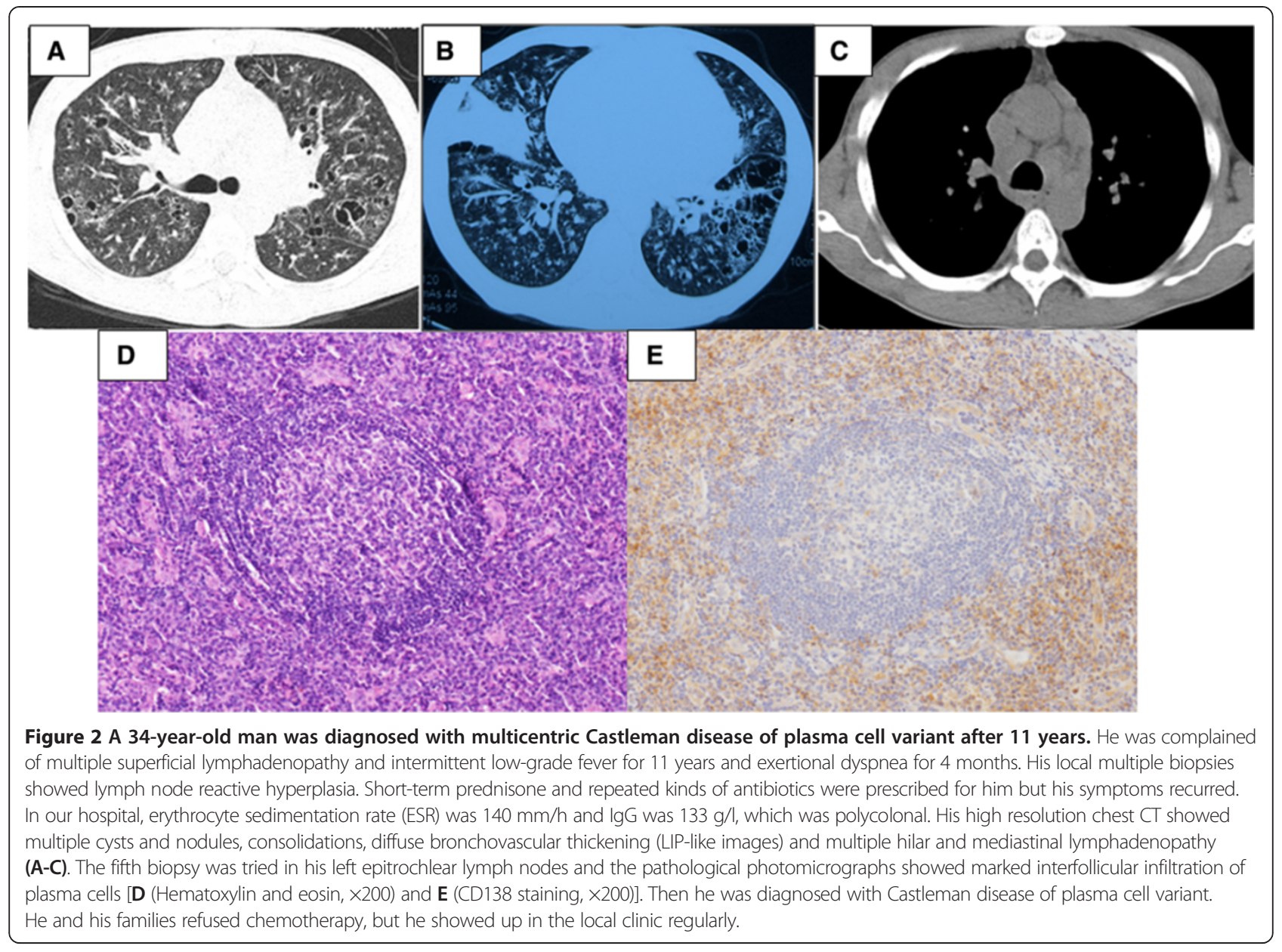

[4-6,23-25], but most of them were focused on the radiologic features, HIV-associated $\mathrm{CD}$ and/or the pulmonary manifestations. Our study is the first of a larger analysis to describe the characteristics of respiratory or intrathoracic $\mathrm{CD}$ in a single tertiary-referral hospital.

As HIV antibody screening was not popular in our hospital before the 21 century, HIV tests were not

\section{Table 3 Surgeries for the enrolled UCDs and MCDs}

\begin{tabular}{lll}
\hline & UCD $(\mathbf{n}=\mathbf{1 6})$ & MCD $(\mathbf{n}=\mathbf{3 2})$ \\
\hline Thoracic surgery & & \\
Open thoracic surgery & $16 / 100 \%$ & 0 \\
VATS $^{\varepsilon}$ & $1 / 16.67 \%$ & $8 / 25 \%$ \\
Abdominal surgery & 0 & $1 / 3.12 \%$ \\
Superficial LN biopsy & 0 & $21 / 65.6 \%$ \\
Cervical LN & 0 & $9 / 28.13 \%$ \\
Axillary LN & 0 & $6 / 18.75 \%$ \\
Supratrochlear LN & 0 & $1 / 3.12 \%$ \\
Percutaneous lung biopsy & 0 & $2 / 6.25 \%$ \\
\hline
\end{tabular}

VATS: video-assisted thoracic surgery LN: Lymphonode.

EOne case with UCD was performed VATS initially, then he was changed to open thoracic surgery because of severe adhesion in the surgery field. available for $6 \mathrm{MCD}$ cases. But all enrolled cases who had HIV antibody screening had negative results. Those without HIV tests were all admitted before 2000, and HIV infection was not so popular in China before 21 century [26]. So, cases enrolled in our study might be considered as a cohort of non-HIV CD.

Forty percent of the $\mathrm{CD}$ in our hospital was located mainly in the thorax, which was less than in previous studies $[4,5]$. In Kwon's study, they included the pathologically diagnosed $\mathrm{CD}$ involving the thoracic cavity, axilla, chest wall, and the lower neck as thoracic CD [6]. The involved location was not analyzed in Shin's [19], Dispenzieri's [1], Robinson's [27] and Waterston's [28] studies.

According to the involved lymph nodal station or organ, $\mathrm{CD}$ was divided into two clinical subtypes, i.e UCD and MCD. Most studies showed that UCD cases were more prominent than MCD cases [6,19,20]. In Dispenzieri's study, $53 \%$ of cases were UCD [1]. But in Kawabata's study, MCD was more common than UCD [29]. And Talat et al. reviewed CD cases reported before September 2009, they found that UCD was more common than MCD in HIV-negative CD groups, but all 
HIV-positive CD cases were MCD [2]. In our study, MCD was more common than UCD. So, the prevalence of different clinical subtypes might vary from different medical centers. And the clinical classification also correlated well with histopathological variants, key clinical features including age, symptoms, treatment strategy and prognosis, and laboratory results, e.g., anemia and serum immunoglobulin levels $[1-3,19,29,30]$, so as our 48 cases with intrathoracic $\mathrm{CD}$. When we compared characteristics between different clinical subtypes of intrathoracic $\mathrm{CD}$ in our study, cases in the MCD group were older than in the UCD group, which was consistent with previous studies [19]. But our MCDs was younger than Dossier's HHV-8 related HIV-negative MCDs, which was suggested to be considered a single clinicopathological entity, regardless of HIV status [31]. As HHV-8 associated tests were not tested for our cases, we could not tell whether this difference was related to HHV-8 infection or not. And there were no gender distribution differences between our UCDs and MCDs.

HV variant cases were more common in our UCD subgroup. Our MCD cases were more symptomic and appeared sicker, including more fever, hepatomegaly and/or splenomegaly and hypoalbuminemia than UCD cases. Our MCD cases showed a lower hemoglobin trend. As there were only 3 UCD cases that had IgG results, we could not compare the IgG difference between the two groups statistically. But $74.1 \%$ of the MCD cases in our group who had Ig serum levels checked showed hyperglobulinemia.

Mass in various locations [4-6,32-34], pleural effusion $[12,35]$, bronchiolitis $[9,14,36]$ and diffuse lung shadows $[6,15,23,24]$ had been reported in cases with intrathoracic $\mathrm{CD}$. Although masses could be found in the hilum [6], mediastinum [6,37-39], intercostal spaces [4,5,40], pleura [12], chest wall [32,33], trachea [8,34], lungs $[6,41]$, et al., most of the reported masses were located in the hilum or mediastinum, and there was only rare case report(s) showing masses in other intrathoracic places. There were no more than 20 reported cases showing masses located in the chest wall [32,33]. No more than 10 reported cases with masses in the intercostal spaces $[4,5,40]$, pleura [12], trachea [8,34], lungs $[6,41]$ respectively. Masses could be seen in both of our UCD and MCD cases. All of our intrathoracic UCD showed solitary masses in the mediastinum, hilum, pluera or lungs. As reported in other studies $[4,6]$, surgical resection was performed for our UCD cases and all of them were alive.

Although all of the MCD cases had lymphadenopathy in the hilum and/or mediastinum, only 3 of them showed a significant mass in the hilum and/or mediastinum. There were no cases in our study which had a mass in the chest wall, intercostal space or trachea.
There were no systemic reviews showing $C D$ cases complicated by pleural effusions. Some rare case reports and the analysis of HIV-infected CD cases showed unilateral or bilateral effusions [12,23,24,35,42]. In the case reports the effusion was exudative [12,35] or chylous [42], but there was no effusion tests for HIV-infected CD cases [23,24]. Lesions originating from or involving the chest wall or pleura, impaired drainage of lymphatic fluids because of the compression caused by CD mass or lymphadenopathy, and/or hypoalbuminia could be the causes of pleural effusions in $\mathrm{CD}$ cases. Effusion was more common in MCD than in UCD: there were 13 cases (40.6\%) with MCD and 2 cases (12.5\%) with UCD which were complicated by pleural effusions. Although most of them had bilateral effusions, most of our MCD showed exudative effusions according to Light's criteria [43]. Without pleural biopsy, we could not explain the causes of effusion.

Follicular bronchiolitis and $\mathrm{BO}$ were the reported bronchiolitis in CD cases $[9,14,36]$. Only Hwangbo et al. reported follicular bronchiolitis in CD cases [14]. But there were some other studies showing the LIP or LIP-like changes in CD cases $[6,23,24]$. Follicular bronchiolitis and LIP were considered to be on the same spectrum of the disease and the distinction is based on the extent and distribution of the lymphocytic infiltration [14].

There was LIP-like changes in our MCD cases' chest CT scans. As there was no lung biopsy for these cases, we could not diagnose them as LIP or follicular bronchiolitis. Most of the $\mathrm{CD}$ cases complicated with $\mathrm{BO}$ had suffered from PNP $[9,36]$, and the pathogenesis of complicated $\mathrm{PNP}$ and $\mathrm{BO}$ was unknown. But the prognosis of CD associated with PNP and BO was poor. Although most of these cases had complete resection of their masses and/or chemotherapy, most of them died of respiratory failure. Our case with MCD associated PNP and $\mathrm{BO}$ died 3 years after thoracic surgery.

Diffuse parenchymal abnormalities had been reviewed by Johkoh et al. [15], Guihot et al. [23,24] and Kwon et al. [6]. In Johkoh's study [15], they analyzed $12 \mathrm{MCD}$ cases who had diffuse lung disease and 7 cases who underwent surgery or transbronchial lung biopsy and were diagnosed with lymphocytic interstitial pneumonitis (LIP). With similar finding in other chest CT images of cases, LIP seemed to present as the pulmonary manifestations of MCD. Guihot et al. [23,24] reviewed pulmonary involvements in HIV-infected CD. They found diffuse bilateral interstitial pneumonia with bronchovascular thickening, septal thickening and subpleural nodules, GGO, and pulmonary consolidations in these cases, without bronchial dilation and cysts. Most of them had a MCD attack, ie, rapid onset and regression within a few days and with a high frequency of relapse. But besides LIP-like images, multiple nodules in different size and 
sites, patchy, GGO, and consolidations were shown in our cases' chest CT scans. On the other hand, some of them had mild symptoms and were stable on medications, so no other therapies were arranged for them. Most of them were stable or improved after therapy.

Our retrospective study had several limitations: First, all enrolled cases had a definite pathological diagnosis, which could result in selection bias. Second, serum Ig analysis was not detected for all enrolled cases, especially for UCD cases. IgG subtype analysis had been performed since 2012 and it was popular after 2013 in our hospital. So, most cases had no initial results of IgG subtype analysis. Third, IgG4-related disease (IgG4-RD) and plasma cell variant $C D$ are especially difficult and often impossible to distinguish based on pathological findings due to their similarities. Serum IL-6 level and HHV-8- related antigen, latency-associated nuclear antigen1, are differential diagnostic factors between IgG4-RD and CD [29]. But all of our cases did not receive tests for HHV-8 and serum interlukin-6 (IL-6) levels, as these items were not carried out in our hospital during that period. Although all of the enrolled cases had no obvious allergic history, which is more common in IgG4-RD patients, some IgG4-RD cases might be enrolled in our cohort. But none of our enrolled cases was revised their diagnosis with IgG4-RD till September 2014.

\section{Conclusions}

MCD was more common than UCD in the intrathoracic $\mathrm{CD}$ cases in our hospital. Intrathoracic MCD was older and more symptomic and presented sicker than UCD cases. HV variant cases were more common in our UCD subgroup. All of our UCD cases showed masses in various intrathoracic locations and surgical resection was performed for all of them and all of them were alive. Masses, pleural effusions, BO, and diffuse pulmonary shadows, including LIP-like images, multiple nodules of different sizes and sites, patchy, GGO, and consolidations were shown in our MCD cases. Most of MCD cases were arranged with chemotherapy and their prognosis were worse than UCD's.

\footnotetext{
Abbreviations

CD: Castleman disease; UCD: Unicentric Castleman disease; MCD: Multicentric Castleman disease; CT: Computed tomography; IgG: Immunoglobin G; LDH: Lactate dehydrogenase; HIV: Human immunodeficiency virus; BO: Bronchiolitis obliterans; LIP: Lymphocytic interstitial pneumonia; GGO: Ground-glass opacity; HV: Hyaline vascular; PC: Plasma cell; PNP: Paraneoplastic pemphigus.
}

\section{Competing interests}

The authors declare that they have no competing interest.

\section{Authors' contributions}

$\mathrm{HH}$ served as the guarantor of the paper, and takes responsibility for the integrity of the work as a whole. JML and SL conceived the study, and participated in its design and coordination. ZJX and YX contributed to the design of the study. $\mathrm{HH}$ and $\mathrm{JML}$ performed the statistical analysis and drafted the manuscript. JC, XK, YLB,REF, CH and YZQ participated in data collection. All authors read and approved the final manuscript.

\section{Acknowledgements}

This work was supported by grants from the National Natural Science Foundation of China [Grant 81170055]. We thank all of the patients and staff for their assistance with this research.

\section{Author details}

'Department of Respiratory Medicine, Peking Union Medical College Hospital, Chinese Academy of Medical Sciences \& Peking Union Medical College, \#1 Shuaifuyuan Street, Dongcheng District, Beijing 100730, China. ${ }^{2}$ Radiological Department, Peking Union Medical College Hospital, Chinese Academy of Medical Sciences \& Peking Union Medical College, \#1 Shuaifuyuan Street, Dongcheng District, Beijing 100730, China. ${ }^{3}$ Pathological Department, Peking Union Medical College Hospital, Chinese Academy of Medical Sciences \& Peking Union Medical College, \#1 Shuaifuyuan Street, Dongcheng District, Beijing 100730, China. ${ }^{4}$ Department of Thoracic Surgery, Peking Union Medical College Hospital, Chinese Academy of Medical Sciences \& Peking Union Medical College, \#1 Shuaifuyuan Street, Dongcheng District, Beijing 100730, China.

Received: 17 October 2014 Accepted: 6 March 2015

Published online: 09 April 2015

\section{References}

1. Dispenzieri A, Armitage JO, Loe MJ, Geyer SM, Allred J, Camoriano JK, et al. The clinical spectrum of Castleman's disease. Am J Hematol. 2012;87:997-1002.

2. Talat N, Schulte KM. Castleman's disease: systematic analysis of 416 patients from the literature. Oncologist. 2011;16:1316-24.

3. Zhu SH, Yu YH, Zhang Y, Sun JJ, Han DL, Li J. Clinical features and outcome of patients with HIV-negative multicentric Castleman's disease treated with combination chemotherapy: a report on 10 patients. Med Oncol. 2013;30:492.

4. Kim JH, Jun TG, Sung SW, Shim YS, Han SK, Kim YW, et al. Giant lymph node hyperplasia (Castleman's disease) in the chest. Ann Thorac Surg. 1995:59:1162-5.

5. Ko SF, Wan YL, Ng SH, Lin JW, Hsieh MJ, Fang FM, et al. Imaging features of atypical thoracic Castleman disease. Clin Imaging. 2004;28:280-5.

6. Kwon S, Lee KS, Ahn S, Song I, Kim TS. Thoracic Castleman disease: computed tomography and clinical findings. J Comput Assist Tomogr. 2013;37:1-8.

7. Burrah R, Deshmane V, Althaf S, Yapamakula S, Kurubabala S, Hanumaiah A, et al. Castleman's disease of the pleura. Gen Thorac Cardiovasc Surg. 2011:59:572-4

8. Yu JY, Oh IJ, Kim KS, Kim YI, Lim SC, Kim YC, et al. Castleman's disease presenting as a tracheal mass. Ann Thorac Surg. 2014;97:1798-800.

9. Nikolskaia OV, Nousari CH, Anhalt GJ. Paraneoplastic pemphigus in association with Castleman's disease. Br J Dermatol. 2003;149:1143-51.

10. Chin AC, Stich D, White FV, Radhakrishnan J, Holterman MJ. Paraneoplastic pemphigus and bronchiolitis obliterans associated with a mediastinal mass: a rare case of Castleman's disease with respiratory failure requiring lung transplantation. J Pediatr Surg. 2001;36:E22.

11. Awotedu AA, Otulana BA, Ukoli CO. Giant lymph node hyperplasia of the lung (Castleman's disease) associated with recurrent pleural effusion. Thorax. 1990;:45:775-6.

12. Ko SF, Ng SH, Hsieh MJ, Lin JW, Huang CC, Lee TY, et al. Castleman disease of the pleura: experience with eight surgically proven cases. Ann Thorac Surg. 2003;76:219-24.

13. Gunluoglu G, Olcmen A, Sokucu SN, Akin H, Dincer I. Intrapulmonarylocated Castleman's disease, which was surgically resected without pulmonary resection. Ann Thorac Cardiovasc Surg. 2011;17:580-3.

14. Hwangbo Y, Cha SI, Lee YH, Lee SY, Seo H, Oh S, et al. A case of multicentric Castleman's disease presenting with follicular bronchiolitis. Tuberc Respir Dis (Seoul). 2013;74:23-7.

15. Johkoh T, Muller NL, Ichikado K, Nishimoto N, Yoshizaki K, Honda O, et al. Intrathoracic multicentric Castleman disease: $\mathrm{CT}$ findings in 12 patients. Radiology. 1998;209:477-81.

16. Iyonaga K, Ichikado K, Muranaka H, Fujii K, Yamaguchi T, Suga M. Multicentric Castleman's disease manifesting in the lung: clinical, radiographic, and pathologic findings and successful treatment with corticosteroid and cyclophosphamide. Intern Med. 2003;42:182-6. 
17. Keller AR, Hochholzer L, Castleman B. Hyaline-vascular and plasma-cell types of giant lymph node hyperplasia of the mediastinum and other locations. Cancer. 1972;29:670-83.

18. Cronin DM, Warnke RA. Castleman disease: an update on classification and the spectrum of associated lesions. Adv Anat Pathol. 2009;16:236-46.

19. Shin DY, Jeon YK, Hong YS, Kim TM, Lee SH, Kim DW, et al. Clinical dissection of multicentric Castleman disease. Leuk Lymphoma. 2011:52:1517-22.

20. Ye B, Gao SG, Li W, Yang LH, Zhao SH, Ma K, et al. A retrospective study of unicentric and multicentric Castleman's disease: a report of 52 patients. Med Oncol. 2010;27:1171-8.

21. Milanovic N, Matkovic S, Ristic D, Jelic S, Petrovic M. Significance of tumor burden, vascular endothelial growth factor, lactate dehydrogenase and beta-2 microglobulin serum levels in advanced diffuse large B cell lymphoma. J BUON. 2012;17:497-501.

22. De Marchi G1, De Vita S, Fabris M, Scott CA, Ferraccioli G. Systemic connective tissue disease complicated by Castleman's disease: report of a case and review of the literature. Haematologica. 2004;89:ECR03.

23. Guihot A, Couderc LJ, Agbalika F, Galicier L, Bossi P, Rivaud E, et al. Pulmonary manifestations of multicentric Castleman's disease in HIV infection: a clinical, biological and radiological study. Eur Respir J. 2005;26:118-25.

24. Guihot A, Couderc LJ, Rivaud E, Galicier L, Bossi P, Oksenhendler E, et al. Thoracic radiographic and $C T$ findings of multicentric Castleman disease in HIV-infected patients. J Thorac Imaging. 2007:22:207-11.

25. McAdams HP, Rosado-de-Christenson M, Fishback NF, Templeton PA. Castleman disease of the thorax: radiologic features with clinical and histopathologic correlation. Radiology. 1998;209:221-8.

26. Sheng $L$, Cao WK. HIV/AIDS epidemiology and prevention in China. Chin Med J (Engl). 2008;121:1230-6.

27. Robinson Jr D, Reynolds M, Casper C, Dispenzieri A, Vermeulen J, Payne K, et al. Clinical epidemiology and treatment patterns of patients with multicentric Castleman disease: results from two US treatment centres. Br J Haematol. 2014;165:39-48.

28. Waterston A, Bower M. Fifty years of multicentric Castleman's disease. Acta Oncol. 2004:43:698-704.

29. Kawabata H, Kadowaki N, Nishikori M, Kitawaki T, Kondo T, Ishikawa T, et al. Clinical features and treatment of multicentric castleman's disease: a retrospective study of 21 Japanese patients at a single institute. J Clin Exp Hematop. 2013;53:69-77.

30. Casper $C$. The aetiology and management of Castleman disease at 50 years: translating pathophysiology to patient care. Br J Haematol. 2005;129:3-17.

31. Dossier A, Meignin V, Fieschi C, Boutboul D, Oksenhendler E, Galicier L. Human herpesvirus 8-related Castleman disease in the absence of HIV infection. Clin Infect Dis. 2013;56:833-42.

32. Kurai M, Kondo R, Kobayashi N, Hyogotani A, Yoshida K, Amano J. Castleman's disease arising from the chest wall. Jpn J Thorac Cardiovasc Surg. 2006;54:555-7.

33. Ueda H, Kouso H, Tsukamoto S. Castleman's disease of the chest wall. J Thorac Oncol. 2009;4:426-8

34. Pham $\Pi$, Harrell JH, Harrell JH, Herndier B, Yi ES. Endotracheal castleman disease: a case report. Chest. 2007;131:590-2.

35. Pinheiro VG, Fernandes GH, Cezar LC, Alves NA, de Menezes DB. Castleman's disease accompanied by pleural effusion. J Bras Pneumol. 2008;34:626-30.

36. Jindal T, Meena M, Kumar A, Khaitan BK. Paraneoplastic pemphigus with Castleman's disease and bronchiolitis obliterans. Pediatr Int. 2011;53:1108-9.

37. Pejaver RK, Watson AH. Castleman's disease. Respir Med. 1994;88:309-11.

38. Gupta NK, Torigian DA, Gefter WB, Marshall MB, Liang H, Carver JR, et al. Mediastinal Castleman disease mimicking mediastinal pulmonary sequestration. J Thorac Imaging. 2005;20:229-32.

39. Slater BJ, Nordin AB, Allen CE, Elghetany MT, Heinle JS, Kim ES. Resection of mediastinal castleman's disease: a case report. Pediatr Hematol Oncol. 2013:30:554-6.

40. Stavridis GT, Lau OJ. Castleman's disease arising from the intercostal space. Eur J Cardiothorac Surg. 1993;7:218-9.
41. Yeh CM, Chou CM, Wong LC. Castleman's disease mimicking intrapulmonary malignancy. Ann Thorac Surg. 2007;84:e6-7.

42. Blankenship ME, Rowlett J, Timby JW, Roth RS, Jones RE. Giant lymph node hyperplasia (Castleman's disease) presenting with chylous pleural effusion. Chest. 1997;112:1132-3.

43. Light RW. The Light criteria: the beginning and why they are useful 40 years later. Clin Chest Med. 2013;34:21-6.

\section{Submit your next manuscript to BioMed Central and take full advantage of:}

- Convenient online submission

- Thorough peer review

- No space constraints or color figure charges

- Immediate publication on acceptance

- Inclusion in PubMed, CAS, Scopus and Google Scholar

- Research which is freely available for redistribution 